\title{
A finite element based micromechanical model to simulate fatigue damage development in open-hole laminated composites
}

\author{
Mohammad Ghalandari*1,2, Hossein Toozandehjani ${ }^{3}$, Ibrahim mahariq ${ }^{4}$ \\ ${ }^{1 *}$ corre sponding: Institute of Re search and Development, Duy Tan University, Da Nang, 550000, Viet Nam \\ ${ }^{2}$ Faculty of Electrical-Electronic Engineering, Duy Tan University, Da Nang 550000, Viet Nam \\ Mohammadghlandari@duytan.edu.vn \\ ${ }^{3}$ Assistant Professor Faculties of Mechanical and Mechatronics Engineering \\ h.toozandehjani@shahroodut.ac.ir \\ ${ }^{4}$ College of Engineering and Technology, Ame rican University of the Middle East, Kuwait \\ Ibrahim.Maharik@aum.edu.kw
}

\begin{abstract}
A micromechanical model is implemented to indicate the progressive fatigue problem of a laminated composite with a central circular hole under fatigue loading based on a finite element model. The mechanical properties of the composite lamina are represented based on the characteristics of the fiber and the matrix through a micromechanics model. An appropriatealgorithm is then adopted to simulate fatigue damage development in the composite lamina. According to this algorithm the stress field of the composite subjected to fatigue load is initially obtained using finite element method. Finally, the predicted results of the stresses in the constituents i.e. fiber and matrix are determined according to the micromechanical bridging model. Finally applying proper damage driving relations leads to damage degree in each element. The proposed model is proven to be successful in the observation of the fatigue behavior with stiffness degradation in each elements of the composite in each cycle. Results are reported and validated using those micromechanical model and experimental data available in the literature.
\end{abstract}

Keywords: progressive fatigue model, micromechanical model, finite element simulation, composite laminate

\section{Introduction}

In recent years advanced composite materials [1,2] are being used increasingly in many engineering applications. The high stiffness to weight ratio [3,4] coupled with the flexibility in the selection of lamination scheme make the laminated composite shells attractive structural components for aerospace, automobile and many other vehicles $[5,6]$.

Employing laminated composites in various structures has motivated researchers to propose innovative algorithms and/or methods to simulate their behaviors during design courses[7-9]. One of 
these main challenges and interests is predicating fatigue life by modeling damage which is created by fatigue as a critical mechanisms of failure for composite materials [10-14] .

Predicting the behavior of composite materials is more complex than homogeneous materials under fatigue load and is controlled by the types of the fiber and matrix, directions and number of fiber layers, and also stress ratio [15]. They are anisotropic and the damage mechanisms like delamination, deboning and breakage of fibers, and matrix cracking [16-19] are highlighted as the main reason of failures in these materials. So, the life assessments models based on the fatigue failure in composite materials can be listed in different classifications likes phenomenological residual stiffness and strength models [20].

Shokrieh and Lessard [21] predicted the composite materials fatigue life under the cyclic loading using their proposed a progressive damage model. So, the laminate fatigue life was represented as number of cycle's relationships. Indeed, the approach is emerged from three major components, these components are based on stress calculations, failure assessments and the rules which specified the degradation of material property. This method will be capable to simulate the residual strength and stiffness and life of arbitrary geometry composite under every cyclic loading. Using the same progressive model, Naderi and Maligno [11] adopted an ABAQUS finite element simulation to estimate the fatigue life of carbon/epoxy laminates with different layup sequences. Later on, Lian and Yao [22] developed a MSC Patran/Nastran finite element analysis to simulate the fatigue damage in composite laminates with an own stiffness degradation model to reduce the elastic constants based on the failure mechanism.

Macro mechanical modeling is common approaches for modeling the failure behavior of composite materials $[23,24]$.These models and its governing effective parameters which related to the material properties can be extracted through unidirectional tests on the fibrous lamina. Because of the fiber and matrix materials stresses and the internal stresses causes, it is not possible to study the constituent fiber or matrix failure causes.

In order to overcome on the latter issue, few researchers have proposed employing fiber and matrix properties to determine the internal stresses in the constituents of the composite subjected to fatigue load in micromechanical scale. Zheng et al. [25] proposed the bridging micromechanical model to estimate fatigue strength of unidirectional composites. Later on, Zabihpour et al. [26] proposed a model to observe the manners of stiffness as well as strength degradations of unidirectional composite under cyclic loading. Shi et al. [27] proposed a damage model to investigate the behavior of reinforced composite under fatigue condition. The damage index for fiber and matrix are extracted according to the law of thermodynamics. Then the stiffness matrix of the lamina is calculated using mechanical properties of fiber and matrix. A multi-scale micro-mechanical model presented by 
Kordkheili et al. [7] which predict behavior of composite structure progressively. In this model the kinetic theory of fracture is used to compute damage parameters of constituent i.e. fiber and matrix under cyclic load.

Progressive damage modeling is a widely used failure analysis technique that has been successfully utilized to study the behavior of composite laminates. In this paper the bridging micromechanical model has been used into the progressive damage model via a suitable numerical solution algorithm to predict fatigue life of central circular open-hole composites. The proposed algorithm predicts the fatigue life for composite structures quantitatively for different stress states. Employing this algorithm, we will be able to investigate damage of the matrix and fiber separately to assess the type of failure i.e. fiber breakage and/or de-bonding. Adopting the numerical scheme based on ABAQUS commercial software gives this capability to the algorithm to guess the position of the failure as the study is done for each element in each increment of solution. For open-hole carbon-fiber/epoxy laminates the damage development is investigated subjected to tension-tension fatigue load.

\section{Model development}

\subsection{Model algorithm}

Figure 1 shows the developed algorithm flow chart to employ progressive fatigue damage model together with micromechanical modeling of fiber and matrix to numerically calculate the fatigue life and damage parameter of laminated composite under fatigue load. 


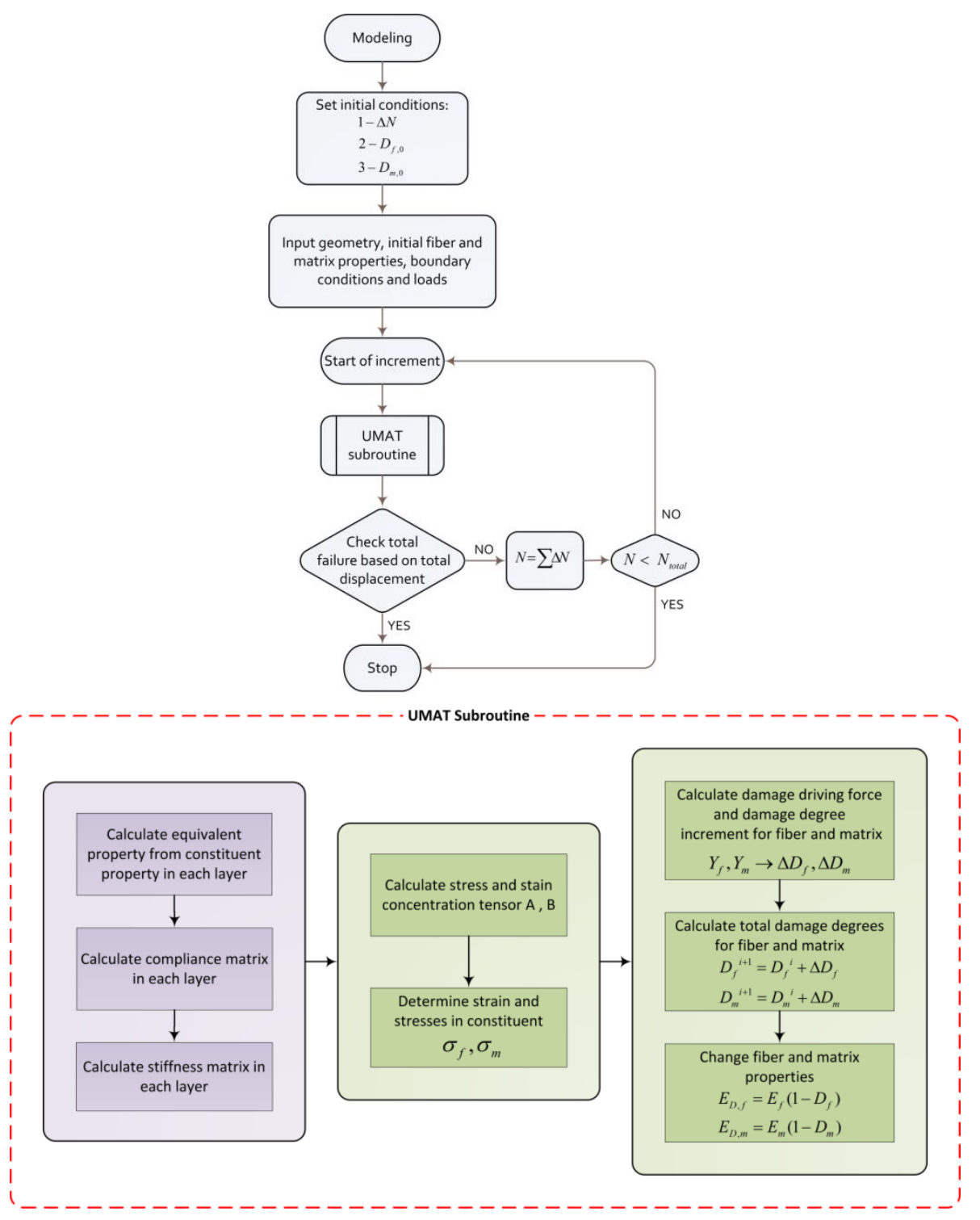

Figure 1. Flowchart of the algorithm

According to this algorithm after set on initial data, the given geometry, material properties of constituents (i.e. the fiber and the matrix) along with fatigue loading is firstly defined. The geometry data like number of layers, thicknesses and fiber directions together with geometry dimensions is employed to model and discretize the composite structure to proper sizes of the elements. Then using fiber volume fraction and properties of the constituents, equivalent property for each element in discretized composite structure is calculated based on rule of mixtures. Now finite element analysis is carried out to calculated element stresses for given cycle of loading. Some transformation matrices should be employed here to transform stresses from global coordinate systems to on-axis coordinat es for all elements. Now using micromechanical relations, the average stresses in the fiber and matrix can be calculated regarding to the average stress of lamina. Then using damage evolution law for fiber and matrix the amount of the damage increment in constituent and consequently total damage 
degrees (failure indices) are calculated for current cycle of loading. In this stage a couple of states may happens.

- Both failure indices are smaller than 1.0. In this situation the solution continues after calculating new fiber and matrix properties.

- Failure index for fiber is still smaller than 1.0 but failure index for matrix exceeds the value 1.0. In this situation we have matrix cracking.

- Failure index for matrix is still smaller than 1.0 but failure index for fiber exceeds the value 1.0. In this situation wehave fiber breakage.

If one of the failure indices reaches 1.0, the material stiffness of relevant constituent is decreased to a quasi-zero number and stiffness properties of composite layer in the finite element model are reconstructed based on new constituent material properties and stress analysis is performed by applying incremental load. Then, if the number of cycles exceeds the predetermined number of total cycles, the program is addressed to terminate. Else, in all plies, material properties change according to the material degradation laws.

Next, the properties of the composite ply are modified by applying the total degree of damage to the fiber and matrix. Then the evaluation on the amount of the stress is conducted again to assess the failure indexes based on the new stresses. The loop is recalculated until a catastrophic failure is indicated or the number of cycles is reached to its maximum values. In case of catastrophic failure, fatigue life and failure mechanisms due to fatigue loading are obtained. The numerical method which is took to estimate the lifecycle of the layer in four steps is summarized as follows:

1) With the transfer of coordinates, the stress is transformed from the $(x, y)$ coordinate to the stress in the $\left(x^{\prime}, y^{\prime}\right)$ coordinate.

2) Calculate average stress in constituent for calculate increment of damage indexes (i.e. $\Delta D_{f}$ and $\left.\Delta D_{m}\right)$

3) Evaluate the new value of the damage indexes in matrix and fiber for cycle $N^{i+1}$ as follows:

$$
\begin{aligned}
& D_{f}{ }^{i+1}=D_{f}{ }^{i}+\Delta D_{f} \\
& D_{m}{ }^{i+1}=D_{m}{ }^{i}+\Delta D_{m}
\end{aligned}
$$

Where $D_{f}{ }^{i+1}$ and $D_{m}{ }^{i+1}$ represented the new degrees of damage in fiber and matrix; respectively.

5) In this step the failure criterion is checked to make sure that new damage indexes are satisfied.

Then, the lamina life is $N=\sum \Delta N$ and if not the above second to the fourth steps are follow ed again until the mentioned failure conditioned meet the criteria. 
This purposed model that defines the damage status at each load cycles is explained by means of a flow chart (Figure 1) which was discussed above and implemented in ABAQUS software as a UMAT subroutine.

\subsection{Micromechanical model}

Indeed, the micromechanical models which are represented here want to extract the composite materials properties based on their constituent properties. Some of these models are as follows: Mixed Role (ROM), Modified Mixed Role and Bridging Model. The bridging model proposed by Huang [25] is based on establishing strain relationships between fibers and matrix. The elastic properties of the composite layer can be obtained in terms of the elastic properties of the fiber and matrix as below.

$$
\begin{aligned}
& E_{11}=V_{f} E_{f}+V_{m} E_{m}, \\
& E_{22}=\frac{\left[V_{f}+V_{m} \eta_{2}\right] E_{f} E_{m}}{V_{m} \eta_{2} E_{f}+V_{f} E_{m}}, \\
& v_{12}=V_{f} v_{f}+V_{m} v_{m}, \text { and } \\
& G_{12}=\frac{\left[V_{f}+V_{m} \eta_{12}\right] G_{f} G_{m}}{V_{m} \eta_{12} G_{f}+V_{f} G_{m}},
\end{aligned}
$$

where the subscript $f$ and $m$ are the indices that represent the fiber and matrix properties, respectively. So, $E_{m}$ and $E_{f}$ are the elastic moduli of fiber and matrix, $v_{f}$ and $v_{m}$ are the Poisson's ratio of fiber and matrix, respectively, $G_{f}$ and $G_{m}$ are the shear moduli of fiber and matrix:

$$
\begin{gathered}
G_{f}=\frac{E_{f}}{2\left(1+v_{f}\right)} \\
G_{m}=\frac{E_{m}}{2\left(1+v_{m}\right)}
\end{gathered}
$$

In the above, $V_{f}$ and $V_{m}=1-V_{f}$ are volume fraction of the fiber and the matrix. In Eq.(2), $\eta_{2}$ and $\eta_{12}$ that obtain experimentally are the correction factors.

The on-axis stresses of the lamina can be obtained from the off-axis stresses using the transformation matrix:

$$
\begin{aligned}
& \sigma_{x^{\prime}}=\sigma_{x} \cos ^{2} \theta+\sigma_{y} \sin ^{2} \theta+2 \tau_{x y} \cos \theta \sin \theta, \\
& \sigma_{y^{\prime}}=\sigma_{x} \sin ^{2} \theta+\sigma_{y} \cos ^{2} \theta-2 \tau_{x y} \cos \theta \sin \theta, \text { and } \\
& \tau_{x^{\prime} y^{\prime}}=-\left(\sigma_{x}-\sigma_{y}\right) \sin \theta \cos \theta+\tau_{x y}\left(\cos ^{2} \theta-\sin ^{2} \theta\right),
\end{aligned}
$$

where $\theta$ is the angle between the natural coordinate system $(x, y)$ and the material coordinate system $\left(x^{\prime}, y^{\prime}\right)$ which is shown in Figure 2. 

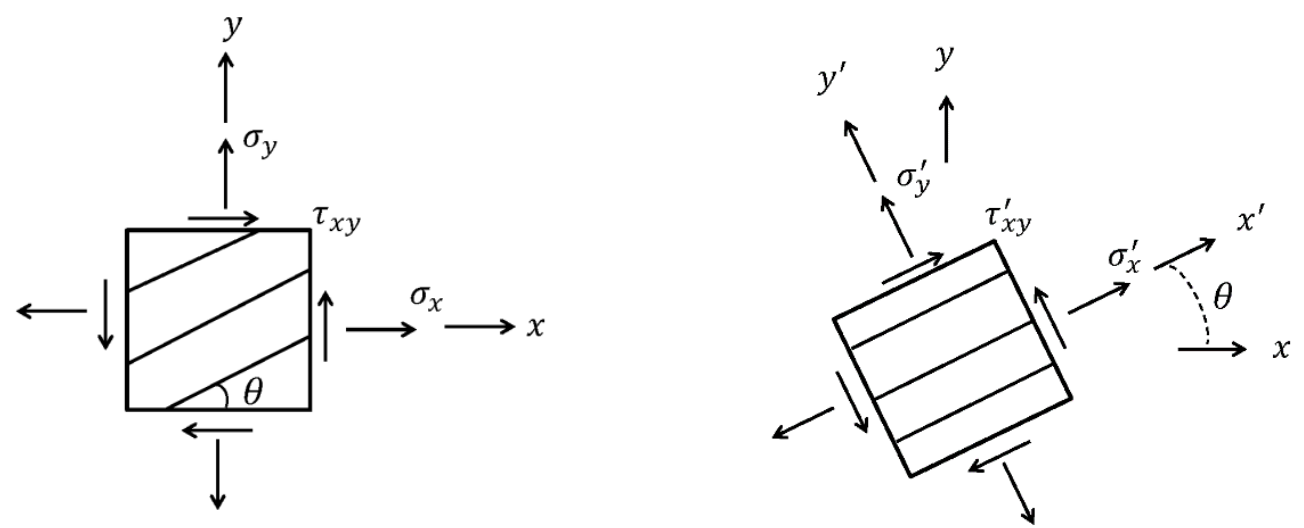

Figure 2. Plane stress transformation

The constitutive equation correlating the stress and strain in a lamina is expressed as

$\{d \varepsilon\}=[S]\{d \sigma\}$

where $S_{i j}$ is the compliance matrix.

It is assumed that the fibers are transversely isotropic thus, the stress and strain relationship are defined as

$$
\left\{d \varepsilon^{f}\right\}=\left[S^{f}\right]\left\{d \sigma^{f}\right\} .
$$

if matrix is isotropic, then

$\left\{d \varepsilon^{m}\right\}=\left[S^{m}\right]\left\{d \sigma^{m}\right\}$.

In the strain-stress equation of the lamina assuming plane stress condition is reasonable and can represented as

$$
\left[S^{k}\right]=\left[\begin{array}{ccc}
\frac{1}{E_{k}} & -\frac{v_{k}}{E_{k}} & 0 \\
-\frac{v_{k}}{E_{k}} & \frac{1}{E_{k}} & 0 \\
0 & 0 & \frac{1}{G_{k}}
\end{array}\right], \quad(k=f, m)
$$

The incremental stresses in the fiber are related to those associated $w$ ith the matrix as bellow

$$
\left\{d \sigma^{m}\right\}=[A]\left\{d \sigma^{f}\right\} \text {. }
$$

The relation between stress increments in the fiber and matrix and overall applied stress increment, $\{d \sigma\}$ is considered via equation $(10)$.

$\left\{d \sigma^{f}\right\}=\left(V_{f}[I]+V_{m}[A]\right)^{-1}\{d \sigma\}$

$\left\{d \sigma^{f}\right\}=[B]\{d \sigma\}$

And

$\left\{d \sigma^{m}\right\}=[A]\left(V_{f}[I]+V_{m}[A]\right)^{-1}\{d \sigma\}$

$\left\{d \sigma^{m}\right\}=[A][B]\{d \sigma\}$. 
So a general form of the bridging matrix is:

$$
[A]=\left[\begin{array}{cccccc}
a_{11} & a_{12} & a_{13} & a_{14} & a_{15} & a_{16} \\
& a_{22} & a_{23} & a_{24} & a_{25} & a_{26} \\
& & a_{33} & a_{34} & a_{35} & a_{36} \\
& & & a_{44} & a_{45} & a_{46} \\
& & & & a_{55} & a_{56} \\
\text { zero } & & & & a_{66}
\end{array}\right]
$$

If the problem is taken as two dimensional the matrix form of $\mathbf{A}$ can be represented as follow

$$
[A]=\left[\begin{array}{ccc}
a_{11} & a_{12} & a_{16} \\
& a_{22} & a_{26} \\
\text { zero } & & a_{66}
\end{array}\right] .
$$

The mechanical properties of fiber and matrix are considered isotropic here. The bridging matrix elements properties are defined as:

$$
\begin{aligned}
& a_{11}=E_{m} / E_{f} \\
& a_{22}=0.5\left(1+E_{m} / E_{f}\right) \\
& a_{66}=0.5\left(1+G_{m} / G_{f}\right) \\
& a_{12}=\left(S_{12}^{f}-S_{12}^{m}\right)\left(a_{11}-a_{22}\right) /\left(S_{11}^{f}-S_{11}^{m}\right),
\end{aligned}
$$

And

$$
\begin{aligned}
& a_{16}=\frac{d_{2} \beta_{11}-d_{1} \beta_{21}}{\beta_{11} \beta_{22}-\beta_{12} \beta_{21}} \\
& a_{26}=\frac{d_{1} \beta_{22}-d_{2} \beta_{12}}{\beta_{11} \beta_{22}-\beta_{12} \beta_{21}},
\end{aligned}
$$

where

$$
\begin{aligned}
& d_{1}=S_{16}^{m}\left(a_{11}-a_{66}\right) \\
& d_{2}=S_{26}^{m}\left(V_{f}+V_{m} a_{11}\right)\left(a_{22}-a_{66}\right)+S_{16}^{m}\left(V_{f}+V_{m} a_{66}\right) a_{12} \\
& \beta_{11}=S_{12}^{m}-S_{12}^{f} \\
& \beta_{12}=S_{11}^{m}-S_{11}^{f} \\
& \beta_{22}=\left(V_{f}+V_{m} a_{22}\right)\left(S_{12}^{m}-S_{12}^{f}\right) \\
& \beta_{21}=V_{m}\left(S_{12}^{f}-S_{12}^{m}\right) a_{12}-\left(V_{f}+V_{m} a_{11}\right)\left(S_{22}^{f}-S_{22}^{m}\right)
\end{aligned}
$$

And

$$
[B]=\left[\begin{array}{ccc}
b_{11} & b_{12} & b_{16} \\
& b_{22} & b_{26} \\
\text { zero } & b_{66}
\end{array}\right]
$$

Where 


$$
\begin{aligned}
& b_{11}=\left(V_{f}+V_{m} a_{22}\right)\left(V_{f}+V_{m} a_{66}\right) / c \\
& b_{12}=-\left(V_{m} a_{12}\right)\left(V_{f}+V_{m} a_{66}\right) / c \\
& b_{16}=\left[\left(V_{m} a_{12}\right)\left(V_{m} a_{26}\right)-\left(V_{f}+V_{m} a_{22}\right)\left(V_{m} a_{16}\right)\right] / c \\
& b_{22}=\left(V_{f}+V_{m} a_{11}\right)\left(V_{f}+V_{m} a_{66}\right) / c \\
& b_{26}=-\left(V_{m} a_{26}\right)\left(V_{f}+V_{m} a_{11}\right) / c \\
& b_{66}=\left(V_{f}+V_{m} a_{22}\right)\left(V_{f}+V_{m} a_{11}\right) / c
\end{aligned}
$$

Where

$$
c=\left(V_{f}+V_{m} a_{11}\right)\left(V_{f}+V_{m} a_{22}\right)\left(V_{f}+V_{m} a_{66}\right)
$$

\subsection{Material Property Degradation}

Under the first cycles of fatigue loading, the strength of the plies can be considered as higher value than the state of stress. Material properties of the laminated composite are degraded by increasing the number of cycles. Eventually, the failure modes of the composite with respect the mechanical properties are determined by the specific number of cycles. The damage degree under the represented loading condition can be specified by characterizing the degradation of materials as follow:

$D=\frac{E-E_{D}}{E}$,

where $E_{D}$ is the damaged Young's modulus. As $E_{D}$ ranges from $E$ to $0, D$ varies between 0 and 1 . From Eq.(20), the elastic constants with damage of the fiber and matrix can readily be written as

$$
E_{k, D}=E_{k}\left(1-D_{k}\right)
$$

Evolutions of the damage equations can be showed to characterize the damage evolution parameter of the fiber and matrix as following:

$$
\begin{aligned}
& \frac{d D_{k}}{d N}=\frac{A_{k} Y_{k}^{B_{k}}}{\left(1-D_{k}\right)^{C_{k}}}, \quad(k=f, m) \\
& \frac{\Delta D_{k}}{}=\frac{A_{k} Y_{k}^{B_{k}}}{\left(1-D_{k}\right)^{C_{k}}} \Delta N, \quad(k=f, m)
\end{aligned}
$$

where $A_{k}, B_{k}$ and $C_{k}$ are the material parameters, $Y_{k}$ is the damage driving forces for the fiber and matrix. Driving force of the damage are presented as

$Y=-\rho \frac{\partial f}{\partial D}$

where $f$ introduce the free energy per unit mass, and $\rho$ is the medium mass density. During the isothermal process the following relation is obtained as:

$\rho f=W=\int \sigma_{i j} d \varepsilon_{i j}$, 
where $\rho f$ is energy per unit volume, and $W$ introduce the strain energy density. So, The strain energy density with damage can be rew ritten as

$W=\frac{1}{E}\left(\frac{1}{1-D}\right)\left[(1+v) \sigma_{i j} \sigma_{i j}-v\left(\delta_{i j} \sigma_{i j}\right)^{2}\right]$.

Thus, the damage driving forces of the fiber and matrix are express as

$Y_{k}=-\frac{\partial W_{k}}{\partial D_{k}}=\frac{W_{k}}{1-D_{k}}, \quad(k=f, m)$

In last equation $D_{f}$ and $D_{m}$ are damage index in fiber and matrix where imply the fiber breakage and matrix cracking respectively.

\section{Results and discussion}

\subsection{Model verification}

In order to validate the results of the present model, experimental data reported by Shi et al. [27] for E-glass/ polyester GT200 are considered.Tthe mechanical properties of fiber and matrix are show $n$ in Table 1 and Damage parameters of evolution equation are listed in Table 2Table 1.

Table 1. Fiber and matrix mechanical properties [27] (volume fraction $V_{f}=52.2 \%$ )

\begin{tabular}{lll}
\hline & Elastic modulus $(G P a)$ & Poisson's ratio $(\%)$ \\
\hline Fiber & 76 & 0.25 \\
Matrix & 3.4 & 0.35 \\
\hline
\end{tabular}

Table 2. Damage parameter of fiber and matrix [27]

\begin{tabular}{ll|ll|l}
\hline & $\mathrm{A}_{\mathrm{i}}$ & $\mathrm{B}$ & $\mathrm{C}_{\mathrm{m}}$ & $\mathrm{D}_{\mathrm{i}, 0}$ \\
\hline Fiber $(\mathrm{i}=\mathrm{f})$ & $1.89969 \times 10^{-7}$ & 3.70585 & 13.7888 & 0.04529 \\
Matrix $(\mathrm{i}=\mathrm{m})$ & $7.11558 \times 10^{-11}$ & 4.50905 & 2.77 .514 & 0.07280 \\
\hline
\end{tabular}

The fatigue life of the lamina for fiber angle $0^{\circ}$ is compared to the experimental ones in Figure 3. From this figure, maximum $1 \%$ difference between the obtained results from represented model and experimental data from literature are shown. 


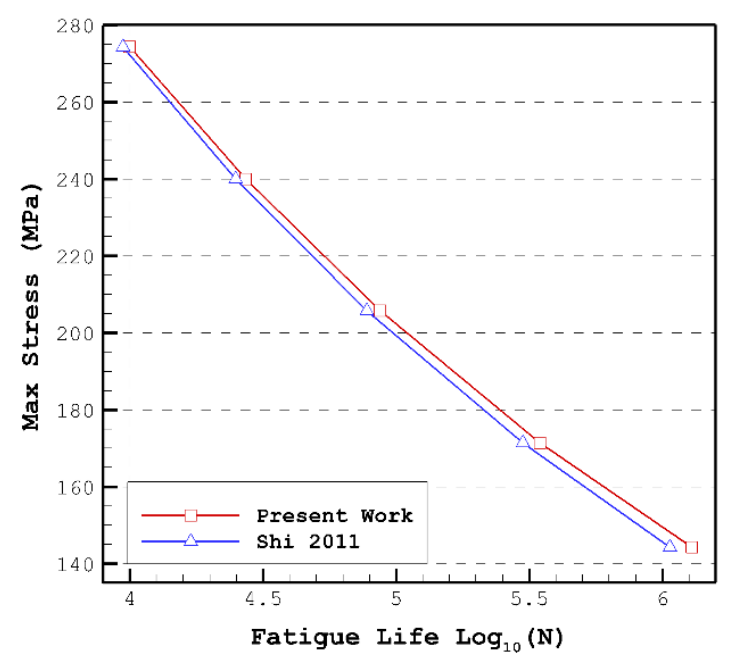

Figure 3. Fatigue lives of the lamina for 0 degree fiber angle under different stress state

Degradation of stiffness and damage degrees of fiber for the UD lamina with 0 and 90 degree of fiber angle under different stress state is illustrated in Figure 4 and Figure 5, respectively. As can be seen stiffness decreases verses number of cycles in a wear out manner.

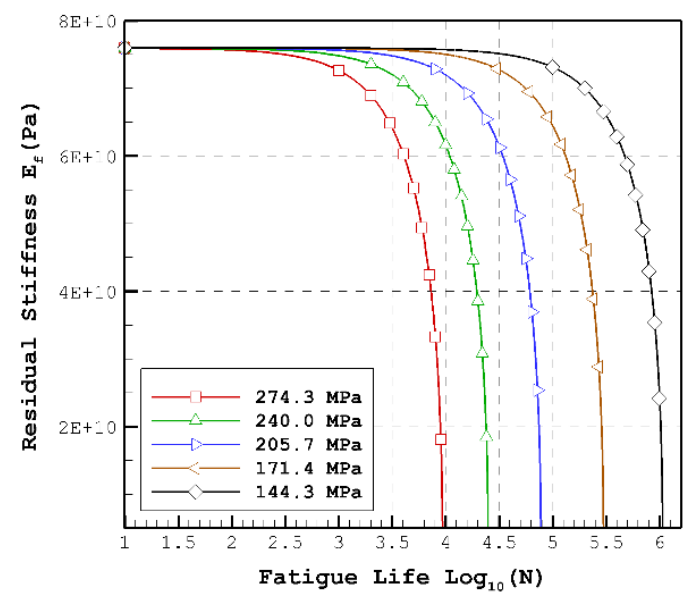

(a)



(b)

Figure 4. a) stiffness degradation and b) Damage degrees of the fiber when fiber angle is 0 under different stress state 


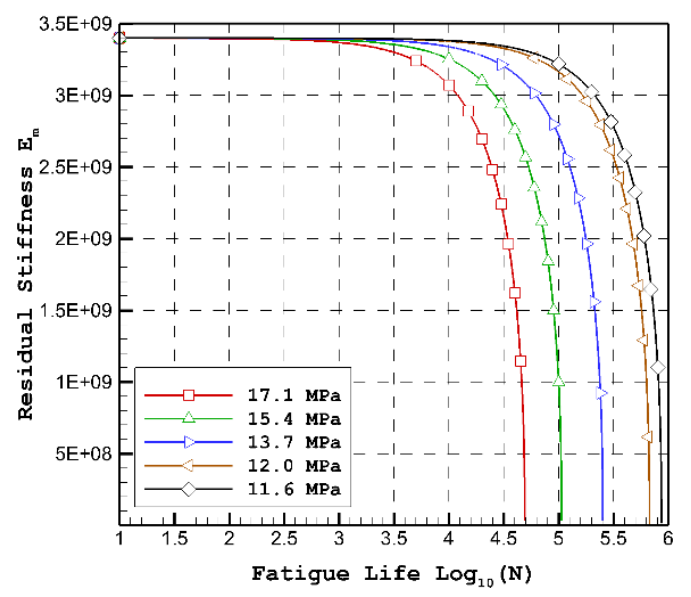

(a)



(b)

Figure 5. a) stiffness degradation and b) Damage degrees of the matrix when fiber angle is 90 under different stress state

\subsection{Model applications}

In order to reveal the application and capability of the presented model to predict the fatigue behavior of lamina composites, an open hole composite lamina (Figure 6) under tension harmonic load with constant stress ratio $\mathrm{R}=0.1$ and $\mathrm{S}_{\max }=200 \mathrm{MPa}$ is considered. Figure 7 and 8 displays distribution of damage index in the fiber phase and matrix phase respectively.

Variation of damage index verses life cycle for element A, B and C are shown in Figure 9, this figure shows that the damage index increases as the number of cycles increases in gradually manner for element $\mathrm{A}$, this increasing for elements $\mathrm{B}$ and $\mathrm{C}$ are more rapidly.

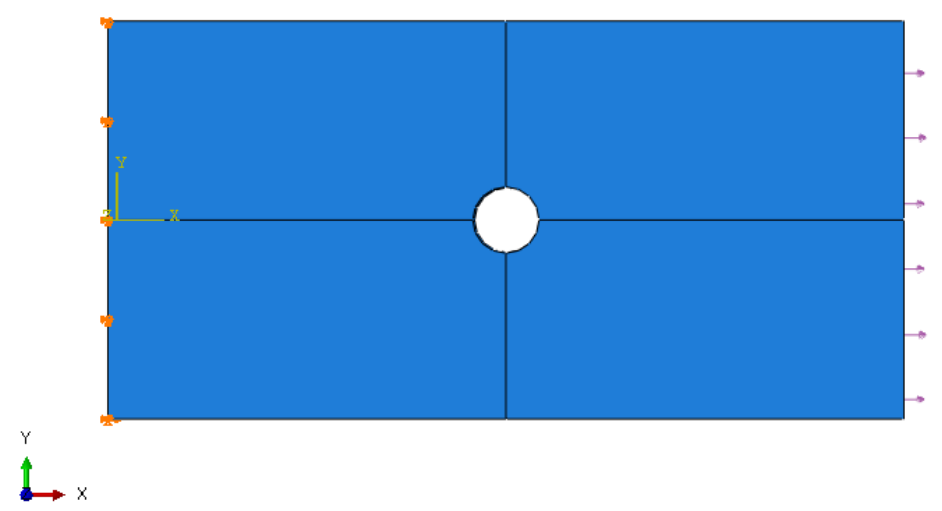

Figure 6. An open hole composite laminate 




Figure 7. Damage index of the fiber $\left(D_{f}\right)$

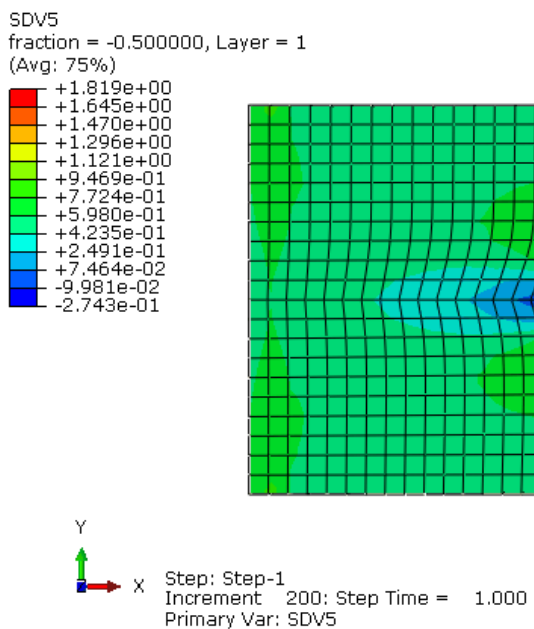

Figure 8. Damage index of the matrix $\left(D_{m}\right)$


Figure 9. Damage degrees verses load cycles of the fiber and matrix for element A, B and C 
The behavior of elastic modulus $E_{11}$ of composite lamina verses number of cycle is shown in Figure 10 , in the damaged element B and C, E11 decreases gradually as the number of cycles increases.
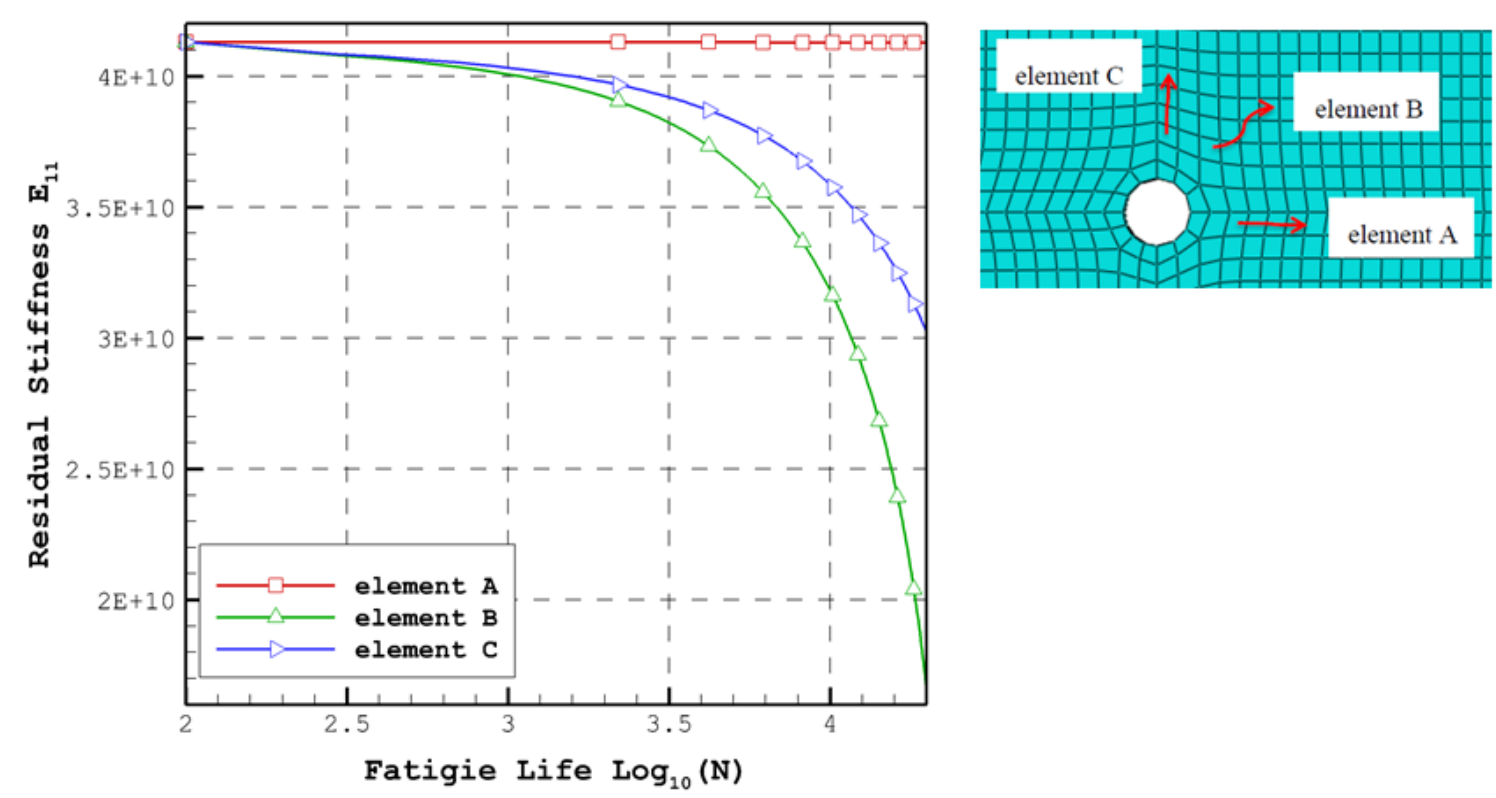

Figure 10. Stiffness reduction verses load cycles for element A, B and C

\section{Conclusions}

In this paper a micromechanical model based on finite element method was proposed and presented comprehensively to study the fatigue life of composite laminates. This micromechanical model used fatigue properties of constituents to expresses fatigue life of composite laminates. The main benefit of this model is to decrease experiments data to predict fatigue life of laminates. A subroutine is developed in the commercial ABAQUS software to implementation of the suggested model. The proposed model will be able to observe the fatigue behavior, stiffness degradation in the composite in each cycle. An acceptable agreement was obtained between the purposed model and experimental results presented in literature. 


\section{References}

1. Thai-Hoang, C.; Nguyen-Thanh, N.; Nguyen-Xuan, H.; Rabczuk, T. An alternative alpha finite element method with discrete shear gap technique for analysis of laminated composite plates. Appl. Math. Comput. 2011,217, 7324-7348.

2. Ghalandari, M.; Ziamolki, A.; Mosavi, A.; Shamshirband, S.; Chau, K.-W.; Bornassi, S. Aeromechanical optimization of first row compressor test stand blades using a hybrid machine learning model of genetic algorithm, artificial neural networks and design of experiments. Eng. Appl. Comput. Fluid Mech. 2019, 13, 892-904.

3. Matbuly, M.S.; Ragb, O.; Nassar, M. Natural frequencies of a functionally graded cracked beam using the differential quadrature method. Appl. Math. Comput. 2009, $215,2307-2316$.

4. Zhang, L.W.; Lei, Z.X.; Liew, K.-M. Computation of vibration solution for functionally graded carbon nanotube-reinforced composite thick plates resting on elastic foundations using the element-free IMLS-Ritz method. Appl. Math. Comput. 2015, 256, 488-504.

5. Katunin, A. Criticality of the self-heating effect in polymers and polymer matrix composites during fatigue, and their application in non-destructive testing. Polymers (Basel). 2019, 11, 19.

6. Sherir, M.A.A.; Hossain, K.; Lachemi, M. Structural performance of polymer fiber reinforced engineered cementitious composites subjected to static and fatigue flexural loading. Polymers (Basel). 2015, 7, 1299-1330.

7. Hosseini Kordkheili, S.A.; Toozandehjani, H.; Soltani, Z. A progressive multi-scale fatigue model for life prediction of laminated composites. J. Compos. Mater. 2017, 51, 2949-2960.

8. Sui, L.; Zhong, Q.; Yu, K.; Xing, F.; Li, P.; Zhou, Y. Flexural fatigue properties of ultrahigh performance engineered cementitious composites (UHP-ECC) reinforced by polymer fibers. Polymers (Basel). 2018, 10, 892.

9. Syaqira, S.; Shamsudin, N.; Leman, Z.; Sapuan, S.M.; Dele-Afolabi, T.T.; Hanim, A.; others Tensile Strength and Moisture Absorption of Sugar Palm-Polyvinyl Butyral Laminated Composites. Polymers (Basel). 2020, 12, 1923.

10. Gemi, L.; Tarakçio \uglu, N.; Akdemir, A.; \cSahin, Ö.S. Progressive fatigue failure behavior of glass/epoxy ( $\$ \backslash \mathrm{pm} \$ 75) 2$ filament-wound pipes under pure internal pressure. Mater. Des. 2009, 30, 4293-4298.

11. Naderi, M.; Maligno, A.R. Fatigue life prediction of carbon/epoxy laminates by stochastic numerical simulation. Compos. Struct. 2012, 94, 1052-1059.

12. Lambert, J.; Chambers, A.R.; Sinclair, I.; Spearing, S.M. 3D damage characterisation and the role of voids in the fatigue of wind turbine blade materials. Compos. Sci. Technol. 2012, 72, 337-343.

13. Böhm Michałand Głowacka, K. Fatigue Life Estimation with Mean Stress Effect Compensation for Lightweight Structures The Case of GLARE 2 Composite. Polymers (Basel). 2020, 12, 251.

14. Hosseini, A.; Ghafoori, E.; Motavalli, M.; Nussbaumer, A.; Zhao, X.-L.; Koller, R. Prestressed unbonded reinforcement system with multiple CFRP plates for fatigue strengthening of steel members. Polymers (Basel). 2018, 10, 264.

15. Benatta, M.A.; Tounsi, A.; Mechab, I.; Bouiadjra, M.B. Mathematical solution for bending of short hybrid composite beams with variable fibers spacing. Appl. Math. 
Comput. 2009, 212, 337-348.

16. Taheri-Behrooz, F.; Shokrieh, M.M.; Lessard, L.B. Residual stiffness in cross-ply laminates subjected to cyclic loading. Compos. Struct. 2008, 85, 205-212.

17. $\mathrm{Wu}, \mathrm{F}$; Y Yao, W. A fatigue damage model of composite materials. Int. J. Fatigue 2010, 32, 134-138.

18. Movaghghar, A.; Lvov, G.I. Theoretical and Experimental Study of Fatigue Strength of Plain Woven Glass/Epoxy Composite. Stroj. Vestnik/Journal Mech. Eng. 2012, 58.

19. Tserpes, K.I.; Papanikos, P.; Labeas, G.; Pantelakis, S. Fatigue damage accumulation and residual strength assessment of CFRP laminates. Compos. Struct. 2004, 63, 219230.

20. Shokrieh, M.M.; Taheri-Behrooz, F. Progressive fatigue damage modeling of cross-ply laminates, I: Modeling strategy. J. Compos. Mater. 2010, 44, 1217-1231.

21. Shokrieh, M.M.; Lessard, L.B. Progressive fatigue damage modeling of composite materials, Part I: Modeling. J. Compos. Mater. 2000, 34, 1056-1080.

22. Lian, W.; Yao, W. Fatigue life prediction of composite laminates by FEA simulation method. Int. J. Fatigue 2010, 32, 123-133.

23. Philippidis, T.P.; Vassilopoulos, A.P. Fatigue of composite laminates under off-axis loading. Int. J. Fatigue 1999, 21, 253-262.

24. Diao, X.; Lessard, L.B.; Shokrieh, M.M. Statistical model for multiaxial fatigue behavior of unidirectional plies. Compos. Sci. Technol. 1999, 59, 2025-2035.

25. Huang, Z.-M. Micromechanical modeling of fatigue strength of unidirectional fibrous composites. Int. J. Fatigue 2002, 24, 659-670.

26. ZABIHPOUR, M.; ADIB, N.S. A micromechanics approach for fatigue of unidirectional fibrous com posites. 2007.

27. Shi, W.; Hu, W.; Zhang, M.; Meng, Q. A damage mechanics model for fatigue life prediction of fiber reinforced polymer composite lamina. Acta Mech. Solida Sin. 2011, $24,399-410$. 